\title{
Long-term outcomes of triangle tilt surgery for obstetric brachial plexus injury
}

\author{
Rahul K. Nath • Xiaomei Liu • Sonya E. Melcher • \\ Jilao Fan
}

Accepted: 14 January 2010/Published online: 4 February 2010

(C) The Author(s) 2010. This article is published with open access at Springerlink.com

\begin{abstract}
Aim The purpose of this study was to evaluate long-term shoulder functional outcomes from a triangle tilt procedure on obstetric patients, who initially presented with medial rotation contracture and scapular deformity secondary to obstetric brachial plexus injury.

Methods We retrospectively studied long-term outcomes both functionally and anatomically in 61 patients (age ranging from 2 to 12 years). Functional movements were evaluated and scored using a modified Mallet scale at different time intervals: preoperatively, 1 year and 2 year following triangle tilt surgery. Shoulder anatomy was examined on radiologic images to evaluate the severity of shoulder deformities preoperatively and anatomical improvement after the surgery.

Results All shoulder functional movements were significantly improved at 1 and 2 year follow-ups. Functional improvements were maintained in shoulder abduction, external rotation and hand-to-mouth movements beyond the first year, and continued in hand-to-neck and hand-tospine movements past 2 years. Remarkable glenohumeral remodeling or reservation of glenoid congruence was observed in all patients over a mean time of 27 months postoperatively.

Conclusion The triangle tilt procedure, which addresses scapular and glenohumeral joint abnormalities characteristic of Erb's palsy, improves shoulder functional movements and anatomical structure in patients over the long-term.
\end{abstract}

R. K. Nath $(\varangle) \cdot$ X. Liu · S. E. Melcher · J. Fan

Texas Nerve and Paralysis Institute, Houston, USA

e-mail: drnath@drnathmedical.com
Keywords Obstetric brachial plexus injury · Triangle tilt surgery · Long-term outcomes . Shoulder deformity

\section{Introduction}

The incidence of obstetric brachial plexus injury in the US is about $0.15 \%$ of live births [1]. The extent of injury includes simple stretch or varying degrees of rupture or avulsion, most commonly of C5 and C6 nerve roots, less likely of the $\mathrm{C} 7$ root, and rarely of $\mathrm{C} 8-\mathrm{T} 1$ roots. About $50 \%$ of patients recover function spontaneously within the first year of life. Patients without adequate recovery of neurological functions tend to develop secondary muscle imbalances and bony deformities at the shoulder joint due to the usually asymmetric injury to the brachial plexus. This asymmetry (with upper roots being injured more frequently than lower roots) leads to twisting deformation of the shoulder joint typically with medial rotation. Clinically, secondary deformities present as contracture of the internal rotators and adductors, glenohumeral dysplasia, humeral head posterior subluxation or dislocation, and/or scapular elevation and rotation in these patients. Secondary deformities are the major cause of long-term morbidity in this patient population [2-5], and limit patients' range of upper extremity motion necessary to perform daily activities of living. Further, posterior humeral subluxation upon the glenoid results in early arthritic changes with pain and loss of function in the teen years [2].

Various treatments, including soft-tissue and bony procedures, have been developed to correct secondary deformities and improve shoulder function. Soft-tissue procedures, such as muscle releases and tendon transfers [6-12], may lead to better shoulder abduction and flexion 
through releasing existing contractures, but does not realign the deformed glenohumeral joint. Derotational osteotomy of the humerus [13-16] as a traditional surgical approach may improve the resting position of the arm, but neglects the glenohumeral deformity, and the scapular elevation and rotation. The recurrence of medial rotation contracture after humeral osteotomy is common due to the unrecognized scapular deformity. Open reduction or arthroscopic anterior capsulectomy alone or with tendon transfers have recently been reported to improve passive external rotation and active range of motion, but results in external rotation deformity with substantially reduced internal rotation postoperatively $[8,18-20]$.

Scapular elevation and rotation has been shown to be one of the major causes of medial rotation contracture in obstetric brachial plexus injury [17, 21]. A unique bony procedure, triangle tilt, to specifically address this scapular deformity, is then indicated. The strategy of the triangle tilt procedure is to solve the impingement of the anteriorly tilted distal acromioclavicular triangle against the humeral head by releasing it from the medial spine of the scapula, and the abnormal twisting of the clavicle by releasing the medial clavicle through osteotomy of the clavicle. This allows the distal triangle to tilt back to its neutral position, reducing the abnormal elevation and rotation of the scapula, therefore, realigning the glenohumeral joint properly.

The purpose of this study was to evaluate long-term shoulder functional outcomes in 61 patients initially presenting with medial rotation contracture and scapular deformities, and treated with the triangle tilt procedure. To do this, functional Mallet movements were compared at different time intervals: preoperatively, 1 year and 2 year following the surgery. Radiologic images were also examined to evaluate the severity of shoulder deformities preoperatively and anatomical improvements after the surgery.

\section{Patients and methods}

Between February 2005 and September 2006, 144 OBPI patients diagnosed with persistent medial rotation contracture and/or scapular deformity, (scapular hypoplasia, elevation and rotation) [17] underwent the triangle tilt surgical procedure [21] developed and performed by the senior author. Sixty-one (42\%) patients over two years of age (to be able to follow Mallet instructions) and having both postoperative 1 year (ranging from 12 to 23 months) and 2 year (over 24 months) follow-ups were included in this study. Among these 61 patients, 24 patients that were evaluated at 1 year follow-up after the triangle tilt procedure have been reported in a previous study [21].
The patients include 30 boys and 31 girls, with 28 leftsided injuries and 33 right-sided injuries. The mean age at the time of triangle tilt surgery was 6 years (ranging from 2 to 12 years). The brachial plexus injuries involved $\mathrm{C} 5-\mathrm{C} 6$ nerve roots in 36 patients, C5-C7 nerve roots in 14 patients, C5-C8 or C5-T1 nerve roots in 11 patients, and the severity of these injuries was equivalent to Narakas' group 3 or 4 [22]. Among these patients, 28 had primary surgery, and the other 33 did not. All 61 had undergone previous secondary muscle surgery, the modified Quad procedure (including a series of tendon transfers, release of latissimus dorsi, teres major, subscapularis, pectoralis major and minor contractures, and neurolysis and decompression of axillary nerve and designed to improve shoulder abduction) [4]. Other secondary procedure, such as posterior glenohumeral capsulorrhaphy (on 14 patients), forearm osteotomy (on one patient) and humeral osteotomy (on four patients), all failed to resolve the shoulder deformity. The medial rotation contracture was still persistent in these patients, even after surgery, physiotherapy and/or splinting.

The triangle tilt procedure is designed to correct bony deformities developed from the initial nerve injury as introduced in a previous study [21]. Briefly, this procedure consists of osteotomy of the clavicle between the proximal and distal thirds, osteotomy of the acromion at its junction with the spine of the scapula, and ostectomy of the superior medial angle of the scapula. A customized splint is then placed with the arm in adduction, neutral position of the humerus with external rotation, and the forearm in full supination. Splinting was maintained for 6 weeks, after which time the splint was only worn at night for an additional 3-6 months. Posterior glenohumeral capsulorrhaphy was performed in patients with frank dislocation of the shoulder diagnosed through preoperative CT or MRI. All the operations were performed by the same surgeon, who has over 12 years of experience with obstetric brachial plexus injury.

Pre- and postoperative shoulder functions of patients were evaluated by the surgeon in the clinic and by trained scientists (independent of the surgeon) from photo/video records, and scored using a modified Mallet scale [23]. These functional movements include shoulder abduction, external rotation, hand-to-mouth movement, hand-to-neck movement, and hand-to-spine movement, which score from 1 (no movement) to 5 (normal movement). In addition to the modified Mallet functional movements, the angle of apparent supination (which is the angle between the midline of the body and the tangential line to the palm with arms straight) was also recorded and measured for each patient in order to define the functional disability more precisely (neutral $=0$, full supination $=90^{\circ}$, full pronation $=-90^{\circ}$ ). 
CT and MRI images were examined to determine the severity of posterior subluxation, glenoid retroversion, and scapular deformity pre- and postoperatively. Posterior subluxation and glenoid retroversion were quantified through axial images (that represent the exact relationship between the humeral head and the glenoid/pseudoglenoid) according to methods modified from Friedman et al. [24] and Water et al. [25]. A scapular line (connecting the medial margin of the scapula and the middle of the glenoid surface) and a glenoid line (tangential to the surface of the glenoid fossa that is articulating with the humeral head) were constructed to define the glenoscapular angle. A total of $90^{\circ}$ was subtracted from this angle to define the degree of glenoid version (normal value $=0^{\circ}$ ). The scapular line was also used to determine posterior subluxation, which is the percentage of the greatest diameter of the humeral head anterior to the scapular line (normal value $=50 \%$ ). Graphic software (Universal Desktop Ruler, AVPSoft.com, Voronezh, Russia) was used for all measurements from radiologic images.
The mean and standard error were determined for each measured parameter. The Wilcoxon signed-rank test was used to compare means of pre- and postoperative parameters. Spearman rank-order test was used to determine the correlation between apparent supination and total modified Mallet score. $p$ values $<0.05$ were considered to be statistically significant. All statistical analyses was performed using Analyse-It plugin (Leeds, UK) for Microsoft Excel 2003 software.

\section{Results}

Patients that were included in this study presented with similar secondary deformities and underwent similar surgical treatments. Functional evaluation was performed at the mean time of 16 months (ranging from 12 to 23 months) for 1 year follow-up and 31 months (ranging from 24 to 43 months) for 2 year follow-up. Modified Mallet functional movements and apparent supination
Fig. 1 Comparisons of individual functional movements of external rotation (a), hand-to-neck (b), handto-spine (c), hand-to-mouth (d), apparent supination (e), and total modified Mallet score (f), in patients with obstetric brachial plexus injury at different time intervals: preoperative, 1 year follow-up and 2 year follow-up. $* p<0.05, * * p<0.01$, $* * * p<0.001$
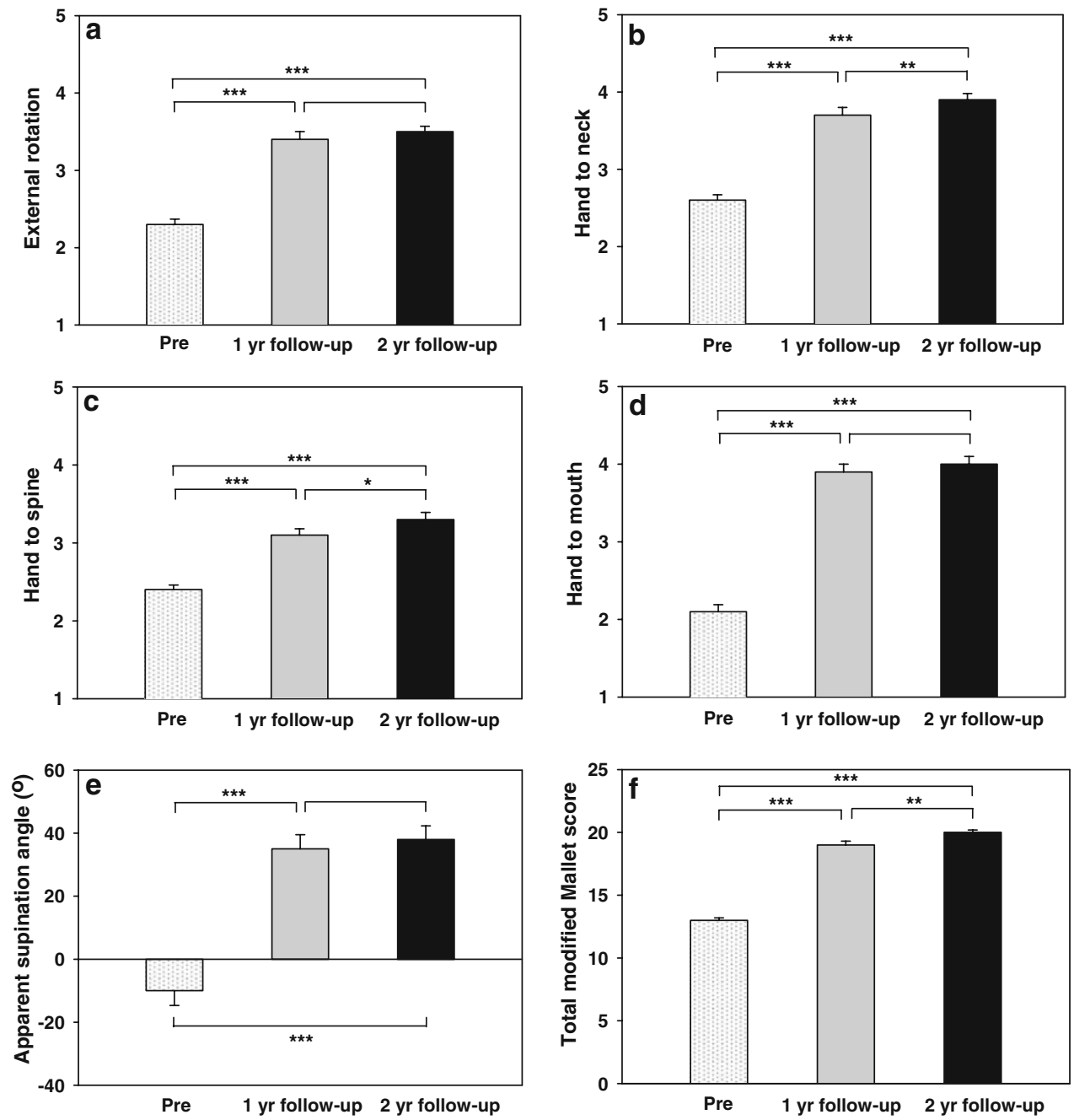
were evaluated for each patient and results are reported in Fig. 1.

Before the triangle tilt surgical procedure, each patient included in this study had undergone a modified Quad procedure [4], which significantly improved the shoulder abduction to a mean score of $4.3 \pm 0.1$ or about $160^{\circ}$. The improvement in shoulder abduction was maintained through the triangle tilt procedure with a mean score of $4.4 \pm 0.1$ at 1 year follow-up and $4.3 \pm 0.1$ at 2 year follow-up.

There were significant differences between preoperative evaluation and 1 year follow-up in the scores for external rotation $(2.3 \pm 0.1$ vs. $3.4 \pm 0.1, p<0.0001)$, hand-toneck $(2.6 \pm 0.1$ vs. $3.7 \pm 0.1, p<0.0001)$, hand-to-spine $(2.4 \pm 0.1$ vs. $3.1 \pm 0.1, \quad p<0.0001)$, hand-to-mouth $(2.1 \pm 0.1$ vs. $3.9 \pm 0.1, p<0.0001)$, total modified Mallet score $(13 \pm 0.2$ vs. $19 \pm 0.3, p<0.0001)$, and apparent supination angle $\left(-10^{\circ} \pm 5^{\circ}\right.$ vs. $\left.35^{\circ} \pm 5^{\circ}, p<0.0001\right)$. There were also significant differences between preoperative evaluation and 2 year follow-up in the above functional movements and total modified Mallet score (all $p$ values < 0.0001). Substantial correlations between apparent supination angle and total modified Mallet score were found at preoperative evaluation, 1 year follow-up and 2 year follow-up $\left(r^{2}=0.6, p<0.0001\right)$. Figures. 2 and 3 show changes in hand-to-mouth and apparent supination postures before and after the surgery from two patients with severe initial medial rotation contracture.

In addition, significant differences were found between 1 year and 2 year follow-ups in the functions of hand-toneck $(3.7 \pm 0.1$ vs. $3.9 \pm 0.1, p=0.003)$, hand-to-spine $(3.1 \pm 0.1$ vs. $3.3 \pm 0.1, p=0.01)$ and total modified Mallet score ( $19 \pm 0.3$ vs. $20 \pm 0.2, p=0.007)$, demonstrating continuing improvements in patient functional recovery. No significant differences were observed between 1 year and 2 year follow-up for the functions of external rotation, hand-to-mouth and apparent supination, demonstrating the stabilization of certain improved functions post-surgically.

From examining preoperative radiologic images, only 6 (10\%) out of 58 patients ( 3 out of 61 patients did not have preoperative exams) had close to normal glenoid anatomy with more than $40 \%$ of the humeral head (Fig. 4, circle) anterior to the scapular line (Fig. 4, dashed arrow). Thirtytwo $(55 \%)$ had a flattened glenoid with progressively posterior subluxation of the humeral head, and $20(35 \%)$ had a biconcave glenoid or pseudoglenoid with less than $10 \%$ of humeral head anterior to the scapular line or
Fig. 2 Comparisons of pre- and postoperative hand-to-mouth movement for patients with obstetric brachial plexus injury. a Preoperative photograph of a 10-year-old boy demonstrating limitation of hand-to-mouth function with typical Bugler's position; b same patient, 24 months after triangle tilt surgery. c Preoperative photograph of a 7-year-old girl demonstrating limitation of hand-to-mouth function; $\mathbf{d}$ same patient, 27 months after triangle tilt surgery
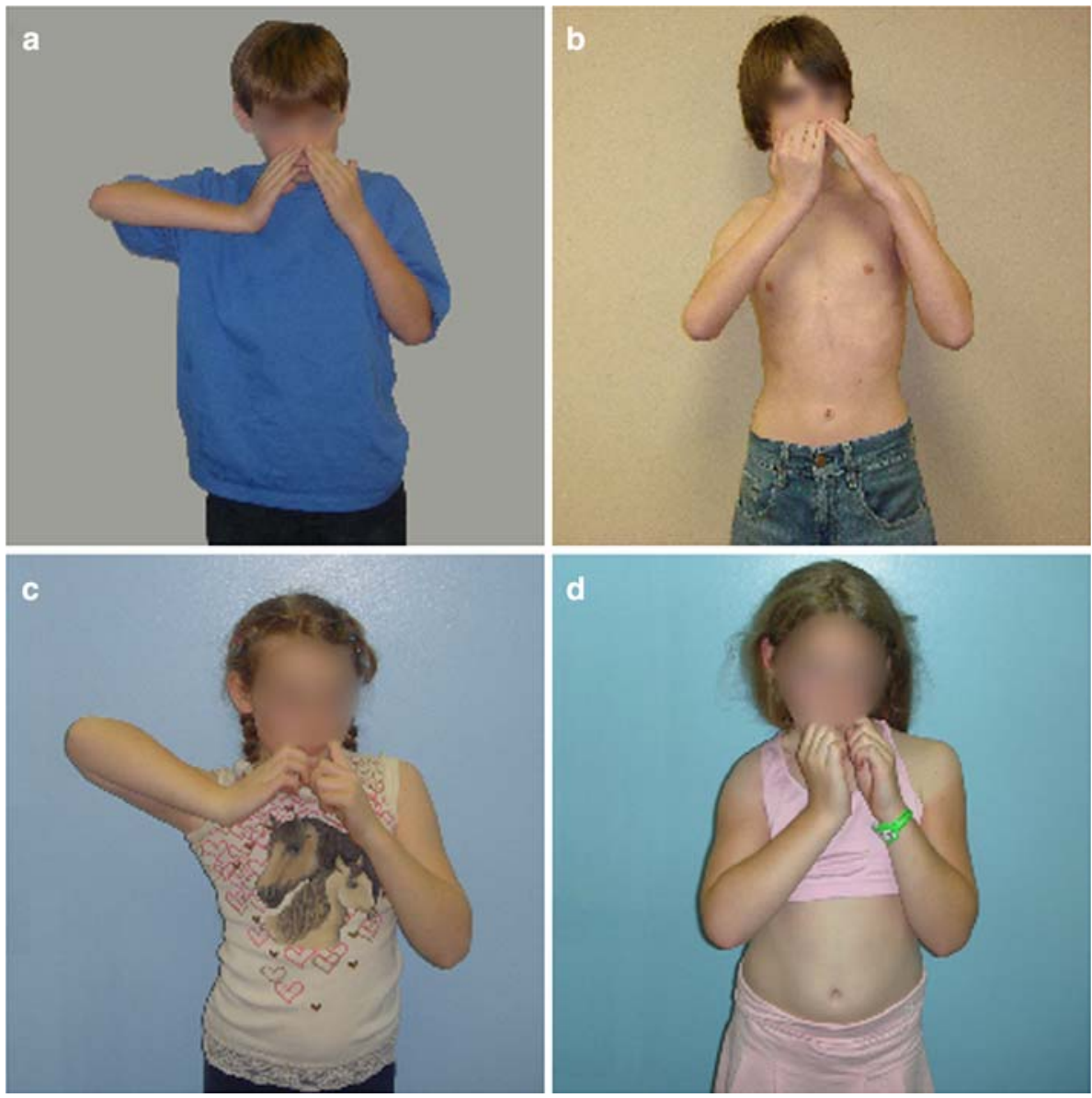
Fig. 3 Comparisons of pre- and postoperative apparent supination movement for patients with obstetric brachial plexus injury. a Preoperative photograph of a 10-year-old boy demonstrating limitation of apparent supination movement; b same patient, 24 months after triangle tilt surgery.

c Preoperative photograph of a 7-year-old girl demonstrating limitation of apparent supination movement; d same patient, 27 months after triangle tilt surgery
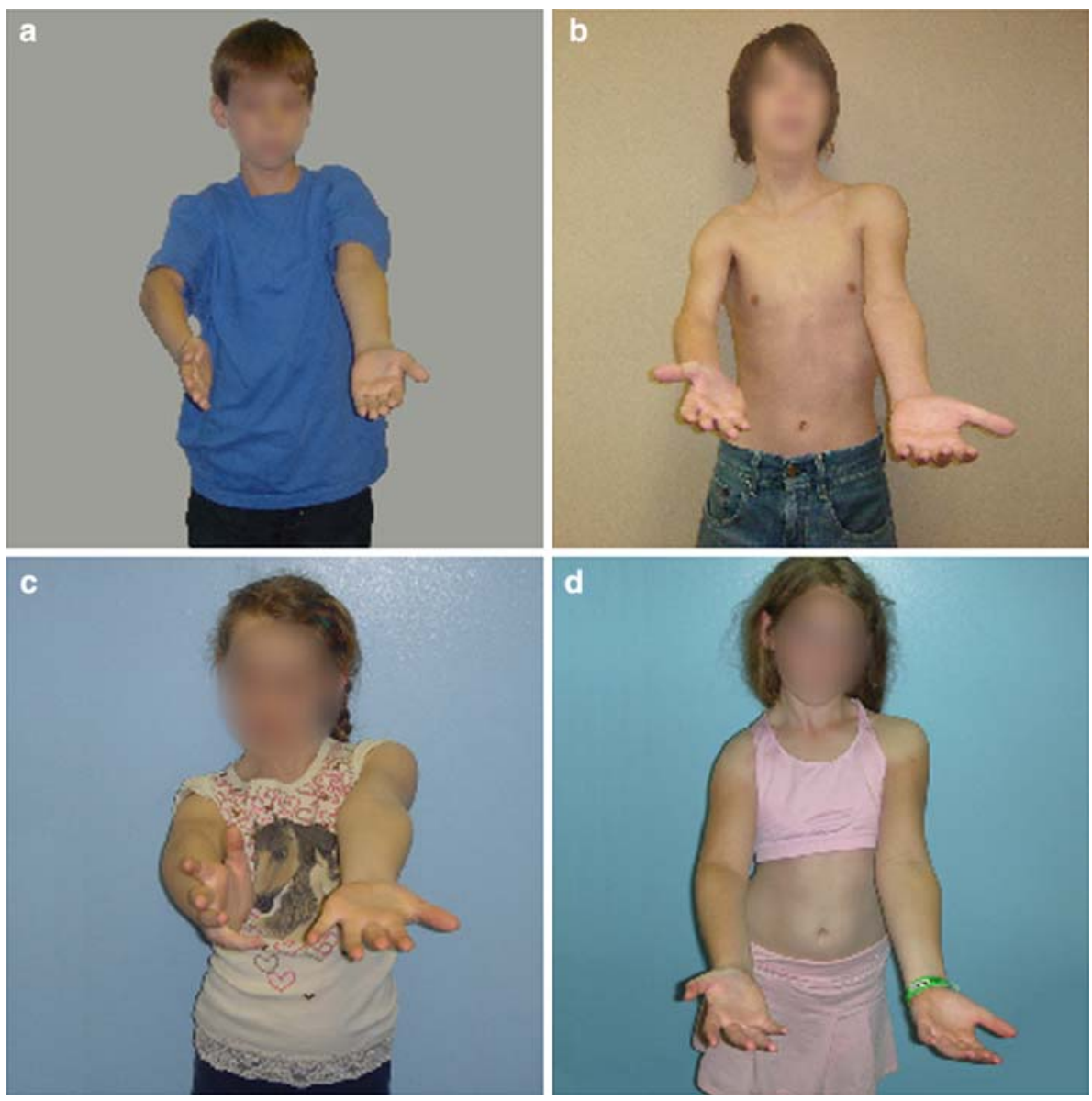

complete dislocation of humeral head. After the mean time of 27 months (ranging from 12 to 49 months), postoperative radiologic images showed that $20(38 \%)$ out of 53 patients ( 8 out of 61 patients did not have postoperative exams) had a normal glenoid, $23(43 \%)$ had a flattened glenoid and only $10(19 \%)$ had a biconcave glenoid or pseudoglenoid. Significant remodeling of the glenohumeral joint was observed. Figure 4 shows changes in glenohumeral joint and scapular elevation before and after the surgery from a patient with initial medial rotation contracture and scapular deformity.

\section{Discussion}

It is well established that abnormal shoulder development of patients suffering from obstetric brachial plexus injury is the most common long-term complication of the initial nerve injury. A variety of surgical procedures have been explored in an effort to treat residual muscle weakness, functional limitation and glenohumeral deformities resulting from insufficient recovery of the original brachial plexus nerve injury. However, there are few procedures having demonstrated long-term improvements both functionally and anatomically in patients with obstetric brachial plexus injury.

Waters and Bae [12] investigated the ability of tendon transfer/lengthening and open reduction to improve shoulder functions and glenohumeral deformity in a cohort of 23 appropriately selected patients with mild glenohumeral deformity. These patients aged from 8 months to 4.2 years with posterior subluxation ranging from 0 to $40 \%$ and glenoid version ranging from $-58^{\circ}$ to $-4^{\circ}$ preoperatively. Their glenohumeral deformity was significantly improved at 11-55 months follow-up. Their total Mallet functional score was also improved from 10 (ranging from 2 to 16 ) to 18 (ranging from 13 to 22). Waters et al. demonstrated the effect of this procedure on treating younger patients with mild deformity. However, no photographic before- and after-evidence was offered, and milder forms of the deformity were addressed, both significant limitations of the study and its applicability to the target population.

Pearl and et al [19] reported an arthroscopic approach to release internal rotation contracture in a group of 33 patients with $45 \%$ having normal or mildly deformed glenoids and 55\% having severely deformed glenoids. Nineteen patients younger than 3 years were treated with an 
a

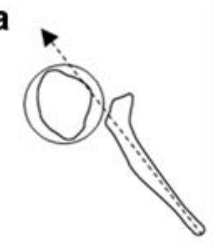

C.
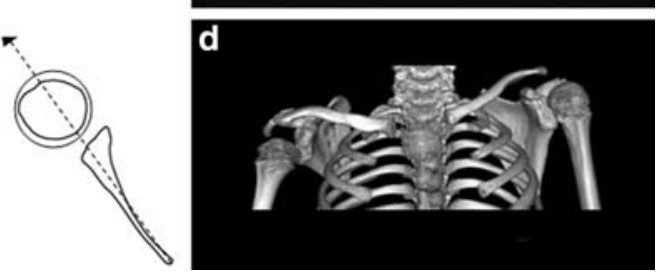

Fig. 4 Comparisons of pre- and postoperative shoulder anatomical structure for a 5-year-old patient with obstetric brachial plexus injury. a Preoperative axial CT diagram demonstrating the right glenohumeral joint deformity (with $-8 \%$ of humeral head anterior to the scapula line and $40^{\circ}$ of glenoid retroversion). b Preoperative 3D CT image demonstrating the right scapular deformity (with $31 \%$ of the scapula above the clavicle relative to the unaffected left scapula). Twenty-six months after the triangle tilt surgery, $\mathbf{c}$ postoperative axial CT diagram demonstrating improvement in the right glenohumeral joint alignment (with $40 \%$ of humeral head anterior to the scapula line and $12^{\circ}$ of glenoid retroversion), and $\mathbf{d}$ postoperative 3D CT image demonstrating reduction in the right scapular elevation (with $5 \%$ of the scapula above the clavicle relative to the unaffected left scapula)

arthroscopic release only, but four had recurrence of the contracture and were treated with repeated arthroscopic release and a latissimus dorsi transfer. Fourteen older patients (ranging from 3 to 12 years) were treated with a combination of arthroscopic release and latissimus dorsi transfer. At 2 year follow-up, all patients showed significantly increased passive external rotation and slightly increased active elevation. No clear functional improvement was obtained. The majority of the patients maintained the concentricity of the glenoid or showed glenohumeral remodeling with a pseudoglenoid only. However, substantially decreased internal rotation was noticed after the surgery, a functional result of surgery probably worse than the initial internal rotation deformity.

Patients in our current study were treated within a relatively wide age range (from 2 to 12 years) with more severe initial deformity of the glenohumeral joint according to the grading criteria from Waters et al. [25] or Pearl et al. [26]. Posterior subluxation ranged from $-60 \%$ to $40 \%$ and glenoid version ranged from $-75^{\circ}$ to $-8^{\circ}$ for affected shoulders preoperatively. After the triangle tilt surgery, patients gained remarkable functional improvements, even for those with more severely deformed glenohumeral joints, which is consistent with our previous study [21]. These functional improvements were maintained over the long-term, and some functions even continued to improve. These patients also showed significant glenohumeral remodeling or reservation of glenoid congruence. No worsening in glenohumeral deformity or functional movements was observed in these patients. This study included four patients who previously had failed derotational humeral osteotomy. The triangle tilt procedure realigned their glenohumeral joints and improved their shoulder functions, which the derotational humeral osteotomy failed to solve.

The patient having the worst glenohumeral deformity in this study was a girl that underwent triangle tilt procedure at 7.1 years old. Her preoperative CT exam showed that her humeral head was completely dislocated posteriorly to the pseudoglenoid (with $-60 \%$ of humeral head anterior to the scapular line and $-75^{\circ}$ of glenoid version). Thirty months after the surgery, her CT images showed significantly improved articulation of the humeral head to the flattened glenoid (with $-5 \%$ of humeral head anterior to the scapular line and $-45^{\circ}$ of glenoid version), which demonstrated significant remodeling of the glenohumeral joint. Her total modified Mallet score was improved from 12 preoperatively to 20 at 2 year follow-up (with both including a score of $5^{\circ}$ or $180^{\circ}$ for shoulder abduction, which was obtained from a modified Quad procedure performed by the surgeon 5 years prior to the triangle tilt procedure). Signs of continuous improvement in both anatomical structure and functional movements were shown at postoperative examination.

Apparent supination was used in this study as a functional evaluator in addition to the Mallet scale-a generally accepted system for the clinical assessment of shoulder functions. The triangle tilt surgery releases the medial rotation contracture through reducing abnormal impingement of the acromioclavicular triangle against the humeral head and scapular elevation/rotation, and puts the upper limb in a more functional position. This is what apparent supination angle measures. Substantial correlation between the apparent supination angle and total modified Mallet score shows that the apparent supination angle can be a good indicator of the functional changes as sensitive as the modified Mallet score.

Comparisons among different procedures are difficult due to inadequate and inconsistent evaluation parameters, as well as to the varied severity of initial nerve injury and subsequent secondary deformities. Furthermore, the majority of papers in this area do not provide pre- and postoperative photographic data sufficiently. Based on the current literature, muscle/tendon transfer with or without open reduction works only on younger patients with a normal to mildly deformed glenohumeral joint, while leaving patients with severe glenohumeral deformity (including complete dislocation) untreatable [6-12]. Arthroscopic anterior capsulectomy may improve certain shoulder functions and/or glenoid remodeling but results in significantly reduced internal rotation, a deformity equal to that being addressed $[18,19]$. More comprehensive and 
long-term outcomes from both functional and anatomical analysis still await [20]. Derotational humeral osteotomy seems to be the main option available to a number of surgeons treating patients ( $>6$ years old) with severe glenohumeral deformity and persistent medial rotation contracture [13-16], even if it does not address the glenohumeral deformity at all and may lead to relatively high recurrence of medial rotation contracture postoperatively.

Affecting factors such as treatment age, severity of secondary deformity and functional limitations are all taken into consideration in the triangle tilt procedure; therefore, it offers a relatively wide treatment range. The triangle tilt procedure alone or combined with the modified Quad procedure (if patients have limited shoulder abduction) serves as an effective approach in the management of patients with secondary deformity from initial brachial plexus injury, which can give patients excellent to outstanding long-term improvements both functionally and anatomically. Ongoing studies comparing pre- and postoperative quality of life for patients undergoing the triangle tilt procedure will add data in evaluating this surgical approach comprehensively.

Open Access This article is distributed under the terms of the Creative Commons Attribution Noncommercial License which permits any noncommercial use, distribution, and reproduction in any medium, provided the original author(s) and source are credited.

\section{References}

1. Foad SL, Mehlman CT, Ying J (2008) The epidemiology of neonatal brachial plexus palsy in the United States. J Bone Joint Surg Am 90:1258-1264

2. Birch R (2001) Late sequelae at the shoulder in obstetrical palsy in children. In: Randelli M, Karlsson J (eds) Surgical techniques in orthopaedics and traumatology: shoulder, Elsevier, Paris, pp 55-200-E-210

3. Kon DS, Darakjian AB, Pearl ML, Kosco AE (2004) Glenohumeral deformity in children with internal rotation contractures secondary to brachial plexus birth palsy: intraoperative arthrographic classification. Radiology 231(3):791-795

4. Nath RK, Paizi M (2007) Improvement in abduction of the shoulder after reconstructive soft-tissue procedures in obstetric brachial plexus palsy. J Bone Joint Surg Br 89(5):620-626

5. van der Sluijs JA, van Ouwerkerk WJ, de Gast A et al (2001) Deformities of the shoulder in infants younger than 12 months with an obstetric lesion of the brachial plexus. J Bone Joint Surg $\mathrm{Br}$ 83(4):551-555

6. Al-Qattan MM (2003) Latissimus dorsi transfer for external rotation weakness of the shoulder in obstetric brachial plexus palsy. J Hand Surg Br 28(5):487-490

7. Pagnotta A, Haerle M, Gilbert A (2004) Long-term results on abduction and external rotation of the shoulder after latissimus dorsi transfer for sequelae of obstetric palsy. Clin Orthop Relat Res 426:199-205

8. van der Sluijs JA, van Ouwerkerk WJ, de Gast A et al (2004) Treatment of internal rotation contracture of the shoulder in obstetric brachial plexus lesions by subscapular tendon lengthening and open reduction: early results and complications. J Pediatr Orthop B 13(3):218-224

9. Safoury Y (2005) Muscle transfer for shoulder reconstruction in obstetrical brachial plexus lesions. Handchir Mikrochir Plast Chir 37(5):332-336

10. Waters PM, Bae DS (2005) Effect of tendon transfers and extraarticular soft-tissue balancing on glenohumeral development in brachial plexus birth palsy. J Bone Joint Surg Am 87(2):320-325

11. El-Gammal TA, Saleh WR, El-Sayed A et al (2006) Tendon transfer around the shoulder in obstetric brachial plexus paralysis: clinical and computed tomographic study. J Pediatr Orthop 26(5):641-646

12. Waters PM, Bae DS (2008) The early effects of tendon transfers and open capsulorrhaphy on glenohumeral deformity in brachial plexus birth palsy. J Bone Joint Surg Am 90(10):2171-2179

13. Kirkos JM, Papadopoulos IA (1998) Late treatment of brachial plexus palsy secondary to birth injuries: rotational osteotomy of the proximal part of the humerus. J Bone Joint Surg Am 80(10):1477-1483

14. Al-Qattan MM (2002) Rotation osteotomy of the humerus for Erb's palsy in children with humeral head deformity. J Hand Surg Am 27(3):479-483

15. Waters PM, Bae DS (2006) The effect of derotational humeral osteotomy on global shoulder function in brachial plexus birth palsy. J Bone Joint Surg Am 88(5):1035-1042

16. Kozin SH (2007) Medial Approach for Humeral Rotational Osteotomy in Children with Residual Brachial Plexus Birth Palsy. Oper Tech Orthop 17(2):88-93

17. Nath RK, Paizi M (2007) Scapular deformity in obstetric brachial plexus palsy: a new finding. Surg Radiol Anat 29:133-140

18. Pearl ML (2003) Arthroscopic release of shoulder contracture secondary to birth palsy: an early report on findings and surgical technique. Arthroscopy 19(6):577-582

19. Pearl ML, Edgerton BW, Kazimiroff PA, Burchette RJ, Wong K (2006) Arthroscopic release and latissimus dorsi transfer for shoulder internal rotation contractures and glenohumeral deformity secondary to brachial plexus birth palsy. J Bone Joint Surg Am 88(3):564-574

20. Pedowitz DI, Gibson B, Williams GR, Kozin SH (2007) Arthroscopic treatment of posterior glenohumeral joint subluxation resulting from brachial plexus birth palsy. J Shoulder Elbow Surg 16(1):6-13

21. Nath RK, Melcher SE, Lyons AB, Paizi M (2007) Surgical correction of the medial rotation contracture in obstetric brachial plexus palsy. J Bone Joint Surg Br 89(12):1638-1644

22. Narakas AO (1987) Obstetrical brachial plexus injuries. In: Lamb DW (ed) The paralysed hand. Churchill-Livingstone, Edinburgh, pp 116-135

23. Mallet J, Mallet J (1972) Obstetrical paralysis of the brachial plexus. II. Therapeutics. Treatment of sequelae. e. Results of different therapeutic techniques and therapeutic indications. Rev Chir Orthop Reparatrice Appar Mot 58(Suppl 1):192-196

24. Friedman RJ, Hawthorne KB, Genez BM (1992) The use of computerized tomography in the measurement of glenoid version. J Bone Joint Surg Am 74(7):1032-1037

25. Waters PM, Smith GR, Jaramillo D (1998) Glenohumeral deformity secondary to brachial plexus birth palsy. J Bone Joint Surg Am 80(5):668-677

26. Pearl ML, Edgerton BW, Kon DS et al (2003) Comparison of arthroscopic findings with magnetic resonance imaging and arthrography in children with glenohumeral deformities secondary to brachial plexus birth palsy. J Bone Joint Surg Am 85(5):890-898 